\title{
Sight and sound of actions share a common neural network
}

\author{
M. A. Giusti, ${ }^{1,2}$ C. Bozzacchi, ${ }^{1,2}$ L. Pizzamiglio ${ }^{1,3}$ and F. Di Russo ${ }^{2,3}$ \\ ${ }^{1}$ Department of Psychology, University of Rome 'La Sapienza', Rome, Italy \\ Department of Education for Motor Activity and Sport, University of Rome 'Foro Italico', Piazza Lauro de Bosis 15, Rome 00135, \\ Italy \\ ${ }^{3}$ Laboratory of Neuropsychology, IRCCS Santa Lucia Foundation, Rome, Italy
}

Keywords: ERPs, human, mirror system, multimodal, repetition suppression

\begin{abstract}
The mirror-neuron system (MNS) connects sensory information that describes an action with a motor plan for performing that action. Recently, studies using the repetition-suppression paradigm have shown that strong activation occurs in the left premotor and superior temporal areas in response to action-related, but not non-action-related, stimuli. However, few studies have investigated the mirror system by using event-related potentials (ERPs) and employing more than one sensory modality in the same sample. In the present study, we compared ERPs that occurred in response to visual and auditory action/non-action-related stimuli to search for evidence of overlapping activations for the two modalities. The results confirmed previous studies that investigated auditory MNS and extended these studies by showing that similar activity existed for the visual modality. Furthermore, we confirmed that the responses to action- and non-action-related stimuli were distinct by demonstrating that, in the case of action-related stimuli, activity was restricted mainly to the left hemisphere, whereas for non-action-related stimuli, activity tended to be more bilateral. The time course of ERP brain sources showed a clear sequence of events that subtended the processing of action-related stimuli. This activity seemed to occur in the left temporal lobe and, in agreement with findings from previous studies of the mirror-neuron network, the information involved appeared to be conveyed subsequently to the premotor area. The left temporo-parietal activity observed following a delay might reflect processing associated with stimulus-related motor preparation.
\end{abstract}

\section{Introduction}

Recently, mounting evidence has indicated that action representations interact with object recognition processes. This also applies to so-called mirror neurons, which respond both when actions are performed and when these actions are seen or heard (Rizzolatti et al., 1996; Decety et al., 1997; Buccino et al., 2001, 2004; Tettamanti et al., 2005; Aziz-Zadeh et al., 2006; Gazzola et al., 2006).

Neurons in the mirror neural system (MNS) have been identified as visual (Gallese et al., 1996), auditory or multimodal (Kohler et al., 2002; Keysers et al., 2003). Despite this, most investigations of the MNS in humans have been restricted to the visual modality. However, two recent studies (Pizzamiglio et al., 2005; Galati et al., 2008) have investigated the cognitive processing of action- vs. non-action-related sounds in the auditory modality using a repetition suppression paradigm (RSP). In these studies, written words were matched to sounds that either were or were not semantically related. When sound processing was compared under congruent and incongruent conditions, the resulting activity was related strictly to semantic congruency and not to the physical differences between the stimuli. Interestingly, in the study by Pizzamiglio et al. (2005), in which event-related

Correspondence: Professor F. Di Russo, ${ }^{2}$ Department of Education for Motor Activity and Sport, as above.

E-mail: francesco.dirusso@uniroma4.it

Received 30 March 2010, revised 31 July 2010, accepted 8 August 2010 potentials (ERPs) were employed, activity related to the recognition of action-related sounds was found in the left premotor cortex and superior temporal area, but not in the right hemisphere. Moreover, as demonstrated by Thierry et al. (2003), bilateral temporal activity was also found for non-action-related stimuli. Galati et al. (2008) have reported similar results using functional magnetic resonance imaging (fMRI) with an identical paradigm. Crucially, these two studies found a clear dissociation between action- and non-action-related activity in a sound recognition task.

A previous fMRI study (Gazzola et al., 2006) also compared responses to the sound of hand actions with responses to the actual execution of hand movements, and, in a separate study (Keysers et al., 2003), compared the sight of different hand actions with hand movements that did not involve objects. The data from these studies provide evidence that the MNS is involved in the recognition of both auditory and visual information concerning action-related stimuli. However, whereas activation was lateralized to the left frontotemporo-parietal areas for auditory stimuli, this activation was bilateral in both visual and motor execution conditions.

The issue of lateralization has been raised by various studies that have investigated the involvement of the human mirror system in the processing of different types of information. In this regard, the left hemisphere, in particular Broca's area, was found to be active in relation to imagery, the observation of motor acts and the processing of sound (Grafton et al., 1996; Iacoboni et al., 1999; Binkofsky et al., 
2000; Buccino et al., 2001; Corballis, 2002; Heiser et al., 2003; Gazzola et al., 2006). Other studies, which used predominantly visual stimuli and motor execution (Binkofsky et al., 1999; Molnar-Szakacs et al., 2005; Aziz-Zadeh et al., 2006), have proposed the existence of a more bilateral mirror system. For example, Iacoboni \& Dapretto (2006) have suggested the existence of a multimodal (visual/auditory) MNS in the left hemisphere, whereas the MNS in the right hemisphere appears to be involved exclusively in visual processing.

The first aim of the present study was to test the degree to which responses to auditory-alone and visual-alone action stimuli share a common cortical network in the MNS that is responsible for processing incoming multimodal information. We hypothesize that the same system processes both visual and auditory action-related information by identifying the shared information involved in an action. In fact, previous studies (Bruzzo et al., 2008) have suggested that the integration of visual, auditory or tactile experience with regard to a motor action is supported by the fact that such experience can activate a 'motor simulation' or motor plan to perform the same action. Furthermore, it has been proposed that MNS activity might even correlate with the ability to anticipate the action or intentions of another person (Kilner et al., 2004). However, there is a lack of conclusive evidence regarding the actual functional role of mirror neurons (Hickok, 2009), especially in relation to action understanding in humans (Lingnau et al., 2009).

To investigate whether audiovisual actions share a common neural structure, we measured the electrophysiological brain activity (namely, ERPs) involved in the processing of action- and non-action-related stimuli for both visual and auditory modalities. The two sets of stimuli utilized corresponded to hand actions and were presented using an RSP.

The second aim of the current study was to determine the dynamics and locations of the auditory mirror neurons in the left precentral and mid-temporal gyri (MTG) (Pizzamiglio et al., 2005; Galati et al., 2008) and the left inferior parietal lobule (Gazzola et al., 2006). Using fMRI, Gazzola et al. (2006) hypothesized that the sensory information processed by the MTG is transmitted subsequently to the parietal and premotor areas, where the corresponding motor representation is activated. Motor plans are then implemented in frontal and parietal structures, whereas temporal lobe activations may reflect the internal generation of auditory and visual correlates of the executed actions. Due to the low temporal resolution of fMRI, the flow of activity within these structures has not yet been clarified. In the present study, ERPs were employed to identify the temporal sequences involved in processing within the mirror system.

\section{Methods}

\section{Subjects}

Fifteen healthy subjects (mean age 23.3 years), four of whom were females, participated in the study. None of the subjects had a history of psychiatric illness or neurological disease; all were right-handed and had normal or corrected-to-normal visual acuity and normal hearing. After the procedure had been explained fully to the subjects, written informed consent was obtained from each participant. The study was approved by the local ethics committee.

\section{Stimuli}

The video and sound clips involved two categories of stimuli, the first of which consisted of human actions such as clapping and banging (action-related category), whereas the second, which did not relate to human actions, consisted of common environmental sounds, such as a telephone ringing and carillon melodies (non-action-related category).

The duration of the clips was fixed at $1000 \mathrm{~ms}$. Video clips were projected towards the centre of a dark screen that subtended to a visual angle of $12.5 \times 10^{\circ}$. Videos that depicted actions were filmed by the researchers involved in the current study. Non-action-related videos were taken from free Internet archives. All video clips were matched for global motion using video editing software and were also matched for luminance. The subjects watched the visual stimuli without sound. Audio clips were monophonic with a frequency of $16 \mathrm{kHz}$, and were also taken from free Internet archives. The audio stimuli were normalized according to the root mean square amplitude. Each stimulus consisted of four different versions so 16 different video and audio clips were employed (see Supporting Table S1). A fixation point was located $1.5^{\circ}$ below each visual stimulus.

\section{Procedure}

We used an RSP, which is capable of isolating the neural correlate of the cognitive response to a stimulus. Repetition suppression refers to a ubiquitous phenomenon in neuroscience where prior processing of a stimulus (or the attributes of the stimulus) leads to a decrease in the degree of activation elicited by processing subsequent stimuli with identical attributes (Henson, 2003; Noppeney \& Penny, 2006). This approach helps to eliminate the confounding variables associated with sensory differences between classes of stimuli that commonly emerge from a direct comparison between different types of tasks, such as the decoding of auditory versus visual action-related stimuli, or between physical different stimuli within the same modality. In this respect, it has been suggested that repetition suppression allows the 'sharpening' of a cortical stimulus representation to occur, i.e. it is a mechanism that produces a sparser but more efficient neural network (e.g. GrillSpector et al., 2006; Gruber et al., 2006).

Figure 1 provides a schematic representation of the experimental design employed in the present study. Subjects were seated in a comfortable chair $114 \mathrm{~cm}$ from the display and were trained to maintain a stable posture and fixation point during the trials, in which pairs of visual and auditory stimuli were presented in rapid succession. No tasks were required of subjects. According to the aforementioned RSP literature, the first stimulus was defined as the 'prime' and the second as the 'target' for each pair of stimuli. Even though no task were required, the second stimuli of the pairs were named targets because they are affected by repetition suppression and are the actual object of the study. Half of the trials were semantically congruent (repeated), i.e. the prime and the target belonged to the same category (e.g. clapping-banging or banging-banging in the action category), whereas the others were 'incongruent' (non-repeated) and consisted of primes and targets in different categories (e.g. carillon-clapping). Supporting Table S1 provides a complete list of prime-target pairs. For each modality, data were recorded in four prime/target conditions: (1) action/congruent, (2) action/incongruent, (3) non-action/congruent and (4) non-action/incongruent. Each stimulus was shown for $1000 \mathrm{~ms}$ and the target was presented $33 \mathrm{~ms}$ after the prime had disappeared; therefore, the duration of each trial was $2033 \mathrm{~ms}$. The inter-trial interval was fixed at $2000 \mathrm{~ms}$. In each run 48 trials were presented and repeated ten times. Rest periods between runs were appropriate to the level of fatigue experienced by the subject. The total duration of the experiment, which included the visual and auditory sessions, was approximately $1 \mathrm{~h}$. Half the subjects began the session with the visual stimuli, whereas the remaining half began with the auditory stimuli. Interviews following the auditory experimental 


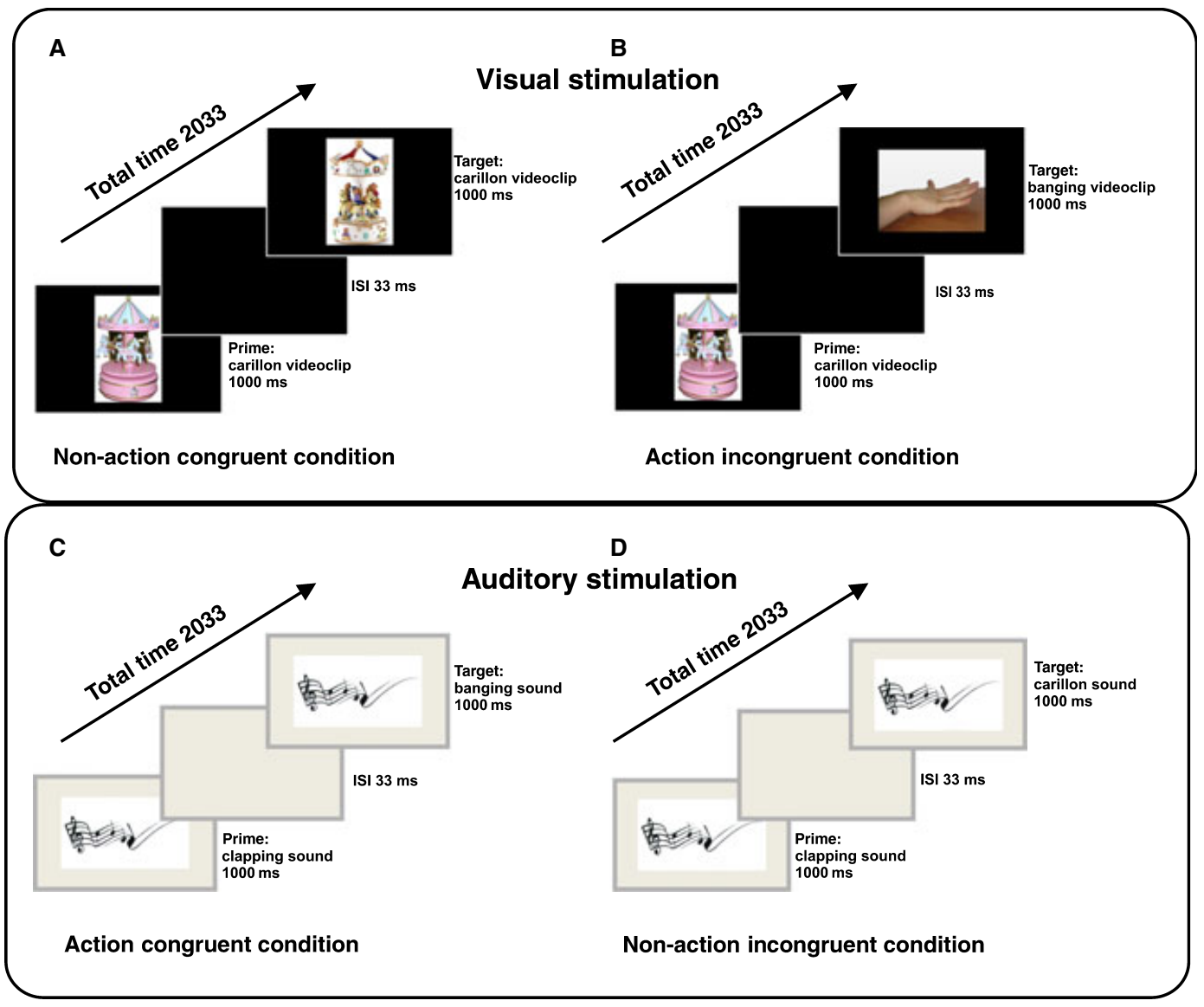

FIG. 1. Schematic representation of the repetition suppression experimental design: stimuli consisted of action-related and non-action-related pairs of video or audio clips presented in separate sessions. Pairs could be semantically congruent (A, C) or incongruent (B, D). Subjects passively listened to or watched the stimuli; no tasks were required. The upper panel represents the visual experiment and the lower panel the auditory experiment.

sessions confirmed that all subjects recognized all the sound stimuli. None of the experimental subjects was excluded from the analysis.

\section{Recording of EEG and data processing}

The electroencephalogram (EEG) was recorded using a BrainVision ${ }^{\mathrm{TM}}$ 64-channel system connected to an active electrode system (ActiCap ${ }^{\mathrm{TM}}$ by Brain Products $\mathrm{GmbH}$, Munich, Germany), which adopted the standard 10-10 system montage (see Di Russo et al., 2002). All scalp channels were referenced to the left mastoid (M1) and then re-referenced to the average reference. Horizontal eye movements were monitored with a bipolar recording from electrodes located at the left and right outer canthi. Blinks and vertical eye movements were recorded by means of an electrode positioned below the left eye and referenced to Fp1. Computerized rejection of artefacts was performed before signal averaging to discard epochs during which deviations in eye position, blinks and amplifier blocking occurred. On average, approximately $20 \%$ of the trials were rejected because they violated these artefact criteria. Blinks were found to be the most frequent cause for rejection. ERPs were obtained by target-only stimulus averaging a minimum of 96 artefact-free trials. The signal was digitized at $250 \mathrm{~Hz}$ and an amplifier band-pass with a range of $0.1-80 \mathrm{~Hz}$ was used along with a $50-\mathrm{Hz}$ notch filter.

The EEG was segmented in all trials for each target stimulus, to give epochs of $1100 \mathrm{~ms}$ (from -100 to $1000 \mathrm{~ms}$ ). The baseline was calculated from -100 to $0 \mathrm{~ms}$ before the prime was shown. ERPs obtained under the congruent and incongruent conditions were compared sample by sample using a running paired $t$-test in the 80-800 ms temporal window to identify any effect of repetition suppression. The onset and peak latency of the major ERPs components were also compared for the two conditions. The alpha level was set at 0.05 , and a Bonferroni correction was employed.

As indicated, and in accordance with the logic of the RSP, in order to isolate the brain area suppressed by repetition, difference waves were obtained by subtracting non-repeated from repeated ERPs. Given that the prime-target repetition was essentially semantic, the difference wave served to isolate the semantic processing of the action- and non-action-related stimuli because only the meaning of the stimulus was repeated. In other words, repeated pairs have the same meaning in terms of action or non-action, despite their physical difference (e.g. clapping-banging). Changes to the strength of the electric field at the scalp were assessed using global field power (GFP; Lehmann \& Skrandies, 1980) for each subject and experimental condition. Values for each point of time were compared using a paired $t$-test.

Three-dimensional (3-D) mapping was used to display the scalp distribution of the ERP data. Given that changes in electric field indicate changes in the underlying generator configuration (Lehmann, 1987), we measured the statistical differences among scalp topographies using a non-parametric randomization test as the topographic analysis of variance (TANOVA) at each time-point with regard to the repeated/non-repeated ERP difference (suppression). For more details on TANOVA, see Murray et al. (2008). Prior to TANOvA, the average ERP segments for all participants were average referenced and transformed to a GFP of 1 , which ensured that the dissimilarity was 
not influenced by higher activity across the scalp in any of the conditions. Thus, this analysis provides a statistical method to determine when and if brain networks that mediate responses to visual and auditory action- or non-action-related stimuli differ.

\section{Source analysis}

The intracranial sources of the differential ERP components were determined using the Brain Electrical Source Analysis system (BESA 2000 version 5.18; MEGIS Software GmbH, Gräfelfing, Germany), which calculated the cerebral areas involved in the task and tested for possible differences in the temporal evolution of the active areas across the conditions studied (action/non-action, visual/auditory). Source analysis was employed to localize the activity in the difference waves (congruent minus incongruent) that were statistically significant.

Initially, the noise-normalized minimum-norm method was employed to estimate the current density dipoles on the cortical scalp. The minimum-norm approach is a method that is used regularly to estimate the distributed electrical current in a brain image at each time of sampling; it is able to resolve the inverse problem without a priori constraints and is also able to reveal the unique constellation of current elements that models the recorded electric field distribution with the smallest amount of overall current (Hämäläinen \& Ilmoniemi, 1984; Ilmoniemi, 1993). The algorithm employed minimizes the source vector current derivate from 1426 evenly distributed dipoles that are located 10 and $30 \%$ below the surface of the brain by using the approach adopted by Dale \& Sereno (1993), in which the correlation between $p_{i}$ of the lead field for regional source $i$ and the inverse of the data covariance matrix is computed together with the largest singular value $\lambda_{\max }$ of the data covariance matrix. The weighting matrix $R$ comprises a diagonal matrix with weights. The equation is: $1 /\left(1+\lambda_{\max }\left(1-p_{i}\right)\right)$. To improve the minimum norm estimate (MNE), we also included depth weighting parameters across the entire source space because, as demonstrated by Lin et al. (2006), depthweighted MNEs can improve the spatial accuracy by allowing displacement errors within $12 \mathrm{~mm}$.

As a second approach, we used spatio-temporal dipole analysis of BESA, which estimates the location and orientation, as well as the time course, of multiple equivalent dipolar sources by calculating the scalp distribution, which is obtained for any given dipole model (forward solution). This distribution was then compared with actual ERPs. Interactive changes in the location and orientation of dipole sources led to the minimization of residual variance between the model and the observed spatio-temporal distribution of ERPs. The position of the electrodes was digitized and averaged across subjects. The 3-D coordinates for each dipole of the BESA model were determined with respect to the Talairach axes and scaled according to brain size. In these calculations, BESA utilized a realistic approximation of the head (which was based on MRI of 24 subjects), and the radius was obtained from the group average $(90 \mathrm{~mm})$. To limit the number of parameters to be estimated, symmetry constraints with regard to location and orientation were applied for each bilateral dipole pair in the scalp distributions that indicated bilateral foci. The possibility that dipoles would interact was reduced by selecting solutions with relatively low dipole moments with the aid of an 'energy' constraint [which was weighted $20 \%$ in the compound cost function as opposed to $80 \%$ for the residual variance (RV)]. The optimal set of parameters was identified in an iterative manner by searching for a minimum in the compound cost function. Dipoles were fitted sequentially. Latency ranges for fitting were chosen (see Results) to minimize overlap among successive, topographically distinct components. To minimize cross-talk and interactions between sources, dipoles that accounted for earlier portions of the waveform were left in place as additional dipoles were added. The dipole fits reported were obtained using the activity peaks of the minimum-norm analysis as starting positions. The goodness of the dipole model was evaluated by measuring its RV as a percentage of the signal variance, as described by the model, and by applying residual orthogonality tests (ROTs; e.g. Bocker et al., 1994). The ROT is based on a Student's $t$-test that measures the sum of cross-products over space and time, and it tests for the presence of systematic spatiotemporal patterns in the residuals of all possible pairs of sub-ensemble averages. A significant positive $t$-value indicates undermodelling, i.e. a failure to describe all systematic variance. Conversely, a significant negative $t$-value indicates overmodelling, i.e. a partial fit to noise. In this study, the ROT was applied by using individual averages as sub-ensemble averages. To decrease the large error variance associated with between-subject ROTs, the residuals were not calculated with respect to the grand average model. Instead, they were calculated after a least-squares fit of the individual time-variant dipole moments. The resulting individual time series for the dipole moments (the source waves) can also be subjected to an orthogonality test, which will be referred to as a source wave orthogonality test (SOT; Bocker et al., 1994). In this procedure, the sum of the cross-products of the source waves is tested for significance over all possible pairs of sub-averages, in a similar way to the residuals in the ROT. A significant positive $t$-value indicates that a proposed source contributes significantly and systematically to potentials recorded at the scalp. A more detailed insight into the temporal dynamics of the source waves can be achieved by calculating SOTs at each point in time separately. In this way, only the reliability of the spatial dimension of the models was evaluated by ROT because the dipole moments were adjusted individually, whereas the interindividual stability of their temporal dynamics was tested by SOT. All $t$-statistics were evaluated for significance at the $5 \%$ level.

\section{Results}

The general shape of the potentials was the same for the two conditions (congruent vs. incongruent). Auditory ERPs included the typical N1, P2 and N2 components (e.g. Teder-Sälejärvi et al., 2002). For the auditory modality, N1 peaked at approximately $120 \mathrm{~ms}$ at the $\mathrm{Cz}$ sensor and $\mathrm{P} 2$ peaked at $190 \mathrm{~ms}$ at the $\mathrm{Fz}$ sensor for the action condition and at $\mathrm{Cz}$ for the non-action condition, whereas N2 peaked at approximately $300 \mathrm{~ms}$ for the Fz sensor (Fig. 2A and B). Visual ERPs also included the typical P1, N1 and P2 components (Di Russo et al., 2002), which peaked at approximately 110 and $160 \mathrm{~ms}$ at the $\mathrm{Oz}$ sensor and at $210 \mathrm{~ms}$ at Pz, respectively (Fig. 2C and D). Peak sensors were used to measure latency and amplitude.

When the latency to onset for the auditory modality was compared between the congruent and incongruent conditions, the P2 and N2 components in the congruent condition were found to be delayed significantly, by approximately 25 and $20 \mathrm{~ms}$, respectively, relative to the incongruent condition $\left(t_{14}>2.56 ; P<0.05\right)$ for both action- and non-action-related stimuli. Differences in peak latency were significant $\left(t_{14}>3.06 ; P<0.025\right)$ only for the P2 component, which was delayed in both the action and the non-action congruent conditions by approximately $20 \mathrm{~ms}$ relative to the incongruent conditions. The onset and peak latency of the auditory N1 component and all the components for the visual modality did not show any significant differences.

With regard to the amplitude of the repetition suppression effect, the difference wave (congruent minus incongruent ERPs) showed a 

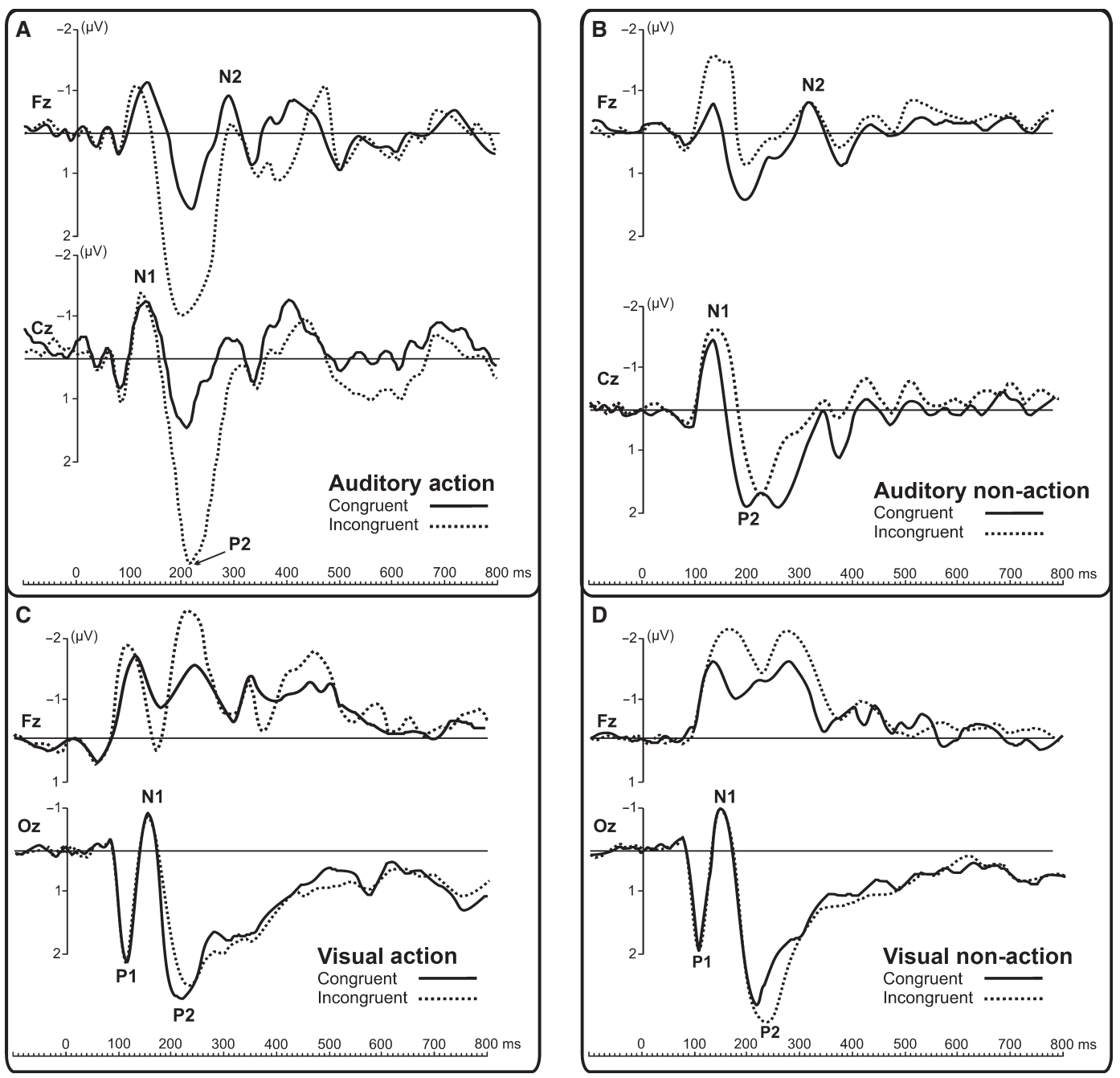

FIG. 2. ERP activity in the congruent (solid line) and incongruent (dotted line) conditions on selected sensors: (A) auditory action-related stimuli; (B) auditory nonaction-related stimuli; (C) visual action-related stimuli; (D) visual non-action-related stimuli. ERP components are labelled at peak amplitude. Note that ERPs arising from congruent (repeated) stimuli were reduced substantially (suppressed) with respect to the incongruent (non-repeated) ERPs.

similar time course for both the visual and the auditory modalities. For action-related stimuli, a negative deflection was present that peaked at the F1 electrode at approximately $170 \mathrm{~ms}$ followed by a peak at $235 \mathrm{~ms}$ at $\mathrm{Fz}$ (negative for the auditory and positive for the visual modality). At $385 \mathrm{~ms}$, a further negative peak at $\mathrm{Fz}$ was observed followed by a positive peak at approximately $470 \mathrm{~ms}$ at $\mathrm{CPz}$ (Fig. 3A). Statistical analyses indicated a significant difference for all these components (see Table 1); this difference was not limited to the peak electrodes and latencies reported here, but also affected adjacent sensors and time frames. Figure $3 b$ shows the differential ERPs elicited by non-action-related stimuli. In this condition, only one positive component was present; it peaked at approximately $160 \mathrm{~ms}$ for both modalities and was statistically significant (see Table 1).

Analysis of the group-averaged global field power waveforms revealed no significant differences between auditory and visual responses (Fig. 4C and D). However, comparison of the action and non-action conditions revealed significant differences with respect to the later action-related components, at 235,385 and $470 \mathrm{~ms}(210$ $245 \mathrm{~ms}, P<0.01$; 380-390 ms, $P<0.05$; 455-480 ms, $P<0.03$ ).

To verify that pairs of stimuli in which the same action was repeated (e.g. clapping-clapping) produced similar suppression as pairs that involved different actions (e.g. clapping-banging), we carried out a further analysis to obtain separate averages for the globally repeated action-related stimuli (same action) and the semantically repeated action-related stimuli (different action). The results indicated that the repetition suppression at approximately $170 \mathrm{~ms}$ was significantly larger for the globally repeated pairs than for the semantically repeated pairs. However, the suppressions at approximately 235, 285 and $470 \mathrm{~ms}$ did not differ between the globally repeated and semantically repeated pairs (data not shown).

Figure 4A shows the 3-D voltage distribution for the components related to the action stimuli. The topography for the first negative components at $170 \mathrm{~ms}$ showed a bilateral distribution in the frontotemporal scalp for the auditory modality and a bilateral distribution in the occipital scalp for the visual modality. At $235 \mathrm{~ms}$ a fronto-medial distribution was present for both auditory and visual modalities but with opposite polarity. For both modalities, the negative component at $385 \mathrm{~ms}$ showed a more anterior fronto-medial distribution than the previous one (at $235 \mathrm{~ms}$ ). Finally, at $470 \mathrm{~ms}$ there was a positive fronto-central distribution and negative activity in the parietal scalp for both modalities.

Figure 4B shows the ERP distribution of the activity at $160 \mathrm{~ms}$ recorded in the non-action-related condition. The scalp distribution 

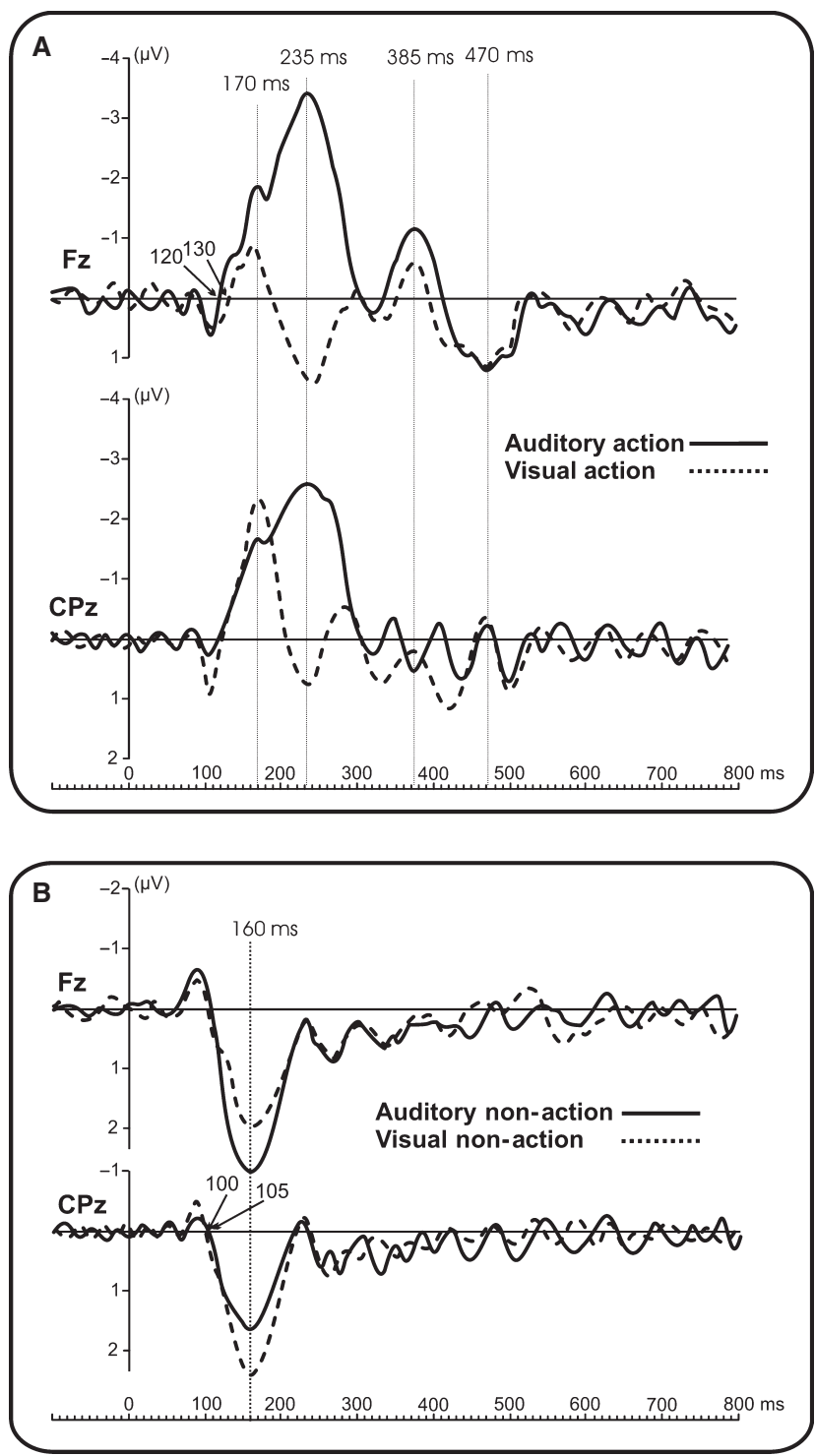

FIG. 3. Differential ERP activity over congruent and incongruent conditions (repetition suppression effect) at the Fz and $\mathrm{CPz}$ electrodes: (A) action-related stimuli for auditory (solid line) and visual (dotted line) modalities, and (B) nonaction-related stimuli for auditory (solid line) and visual (dotted line) modalities. Significant differential activity is marked at peak latencies. Onset of the repetition suppression effect is also indicated by arrows.

TABLE 1. Statistical analysis of the repetition suppression effect for visual and auditory modalities for action- and non-action-related stimuli $($ d.f. $=14)$

\begin{tabular}{lllllll}
\hline & & \multicolumn{2}{l}{ Visual } & & \multicolumn{2}{l}{ Auditory } \\
\cline { 6 - 7 } $\begin{array}{l}\text { Latency } \\
\text { window (ms) }\end{array}$ & $\begin{array}{l}\text { Peak } \\
\text { electrode* }\end{array}$ & $t>$ & $P<$ & & $t>$ & $P<$ \\
\hline Action & & & & & \\
$165-180$ & $\mathrm{Fz}$ & -3.63 & 0.01 & & -3.12 & 0.01 \\
$225-245$ & $\mathrm{Fz}$ & -5.07 & 0.01 & -5.12 & 0.01 \\
$340-360$ & $\mathrm{Fz}$ & -1.99 & 0.05 & -1.99 & 0.05 \\
$460-480$ & $\mathrm{FCz}$ & 1.93 & 0.05 & -2.03 & 0.05 \\
Non-action & & & & & \\
$150-170$ & $\mathrm{~F} 1$ & 2.69 & 0.01 & 2.48 & 0.05 \\
\hline
\end{tabular}

*Sensor where largest amplitude was recorded for each component. was very different for the two modalities. For the auditory modality, a positive activity was present in the medial fronto-temporal area and a negative distribution in the bilateral posterior-temporal areas. In contrast, the visual modality produced positive activity in the medial parieto-occipital area.

With regard to topographical differences, the results of TANOVA for the auditory vs. visual topographies (vertical bars in Fig. 4C and D) showed that, for action-related stimuli, only the scalp distribution at $170 \mathrm{~ms}$ differed significantly $(160-180 \mathrm{~ms}, P<0.01)$. For the nonaction-related stimuli, only the scalp distribution at approximately $160 \mathrm{~ms}$ showed a significant difference $(150-180 \mathrm{~ms}, P<0.02)$.

Source localization obtained by minimum-norm analysis revealed that, for action-related stimuli in the auditory modality (Fig. 5A), the first negative component at $170 \mathrm{~ms}$ appeared to be related to specific sensory processes, because it was localized predominantly to the anterior part of the superior temporal gyrus (STG) close to the lateral fissure. This activity was also stronger in the left hemisphere. Furthermore, at this latency, the activity spread dorsally and bilaterally along the precentral sulcus. For the visual modality (Fig. 5B), at a latency of $170 \mathrm{~ms}$, activity was found to be situated bilaterally in the temporal-occipital areas. For both modalities, the differential activity at $235 \mathrm{~ms}$ was localized mainly around the STG, with stronger lateralization in the left hemisphere. At $385 \mathrm{~ms}$, activity was localized more anteriorly with involvement of the left prefrontal areas. Finally, at $470 \mathrm{~ms}$, activity was localized mainly in the left prefrontal and temporal-parietal areas for both modalities.

The localization of the source of the non-action-related stimuli (Fig. 5C and D) was very different in the two modalities: in the auditory modality (Fig. 5C), activity was localized in the bilateral auditory areas around the superior and middle temporal gyri, whereas in the visual modality (Fig. 5D), activity was localized in the bilateral striate and extrastriate visual areas.

In the case of the spatiotemporal dipole model, the fitting strategy was as follows: for action-related conditions, and taking into account the bilateral scalp distribution (Fig. 4A), bilateral mirrored symmetric dipole pairs were fitted to the component at $170 \mathrm{~ms}$ using the peak activity of the minimum-norm in the lateral fissure as the starting location for the auditory modality and that in the temporal-occipital area for the visual modality. In both modalities a single dipole was then fitted at $235 \mathrm{~ms}$ using the peak activity of the minimum-norm in the left STG as the starting location. At $385 \mathrm{~ms}$, a single dipole was fitted using the peak activity of the minimum-norm in the left prefrontal areas as the starting location. Finally, at $470 \mathrm{~ms}$, a single dipole was fitted using the peak activity of the minimum-norm in the left parietal area as the starting location. For both modalities, the models accounted for more than $97 \%$ of the variance $(\mathrm{RV}>3 \%$ ) in scalp voltage topography over the time range $170-470 \mathrm{~ms}$, which indicated that the models were robust and sufficiently explain the data (e.g. Di Russo et al., 2002). For ROT, none of the channels pointed to a significant residual signal, which indicated that there was no failure in any channel associated with the model. For SOTs, the sources fail to reach significance over the whole interval of fit; this indicates that the dipole moments do not show systematic time courses.

Figure 6A shows the results from the spatiotemporal source models for the action-related conditions. The left panel sets out the time course for the cortical sources that can be seen localized in the right panel. With respect to the auditory modality, the first component at $170 \mathrm{~ms}$ was localized bilaterally within the temporal lobe around the anterior part of the MTG. With respect to the visual modality, the activity at the same latency was localized bilaterally in the occipital lobe within the occipital gyrus. The time course of these sources (averaged over both hemispheres) was quite similar, with a peak at 100 and $150 \mathrm{~ms}$, which 

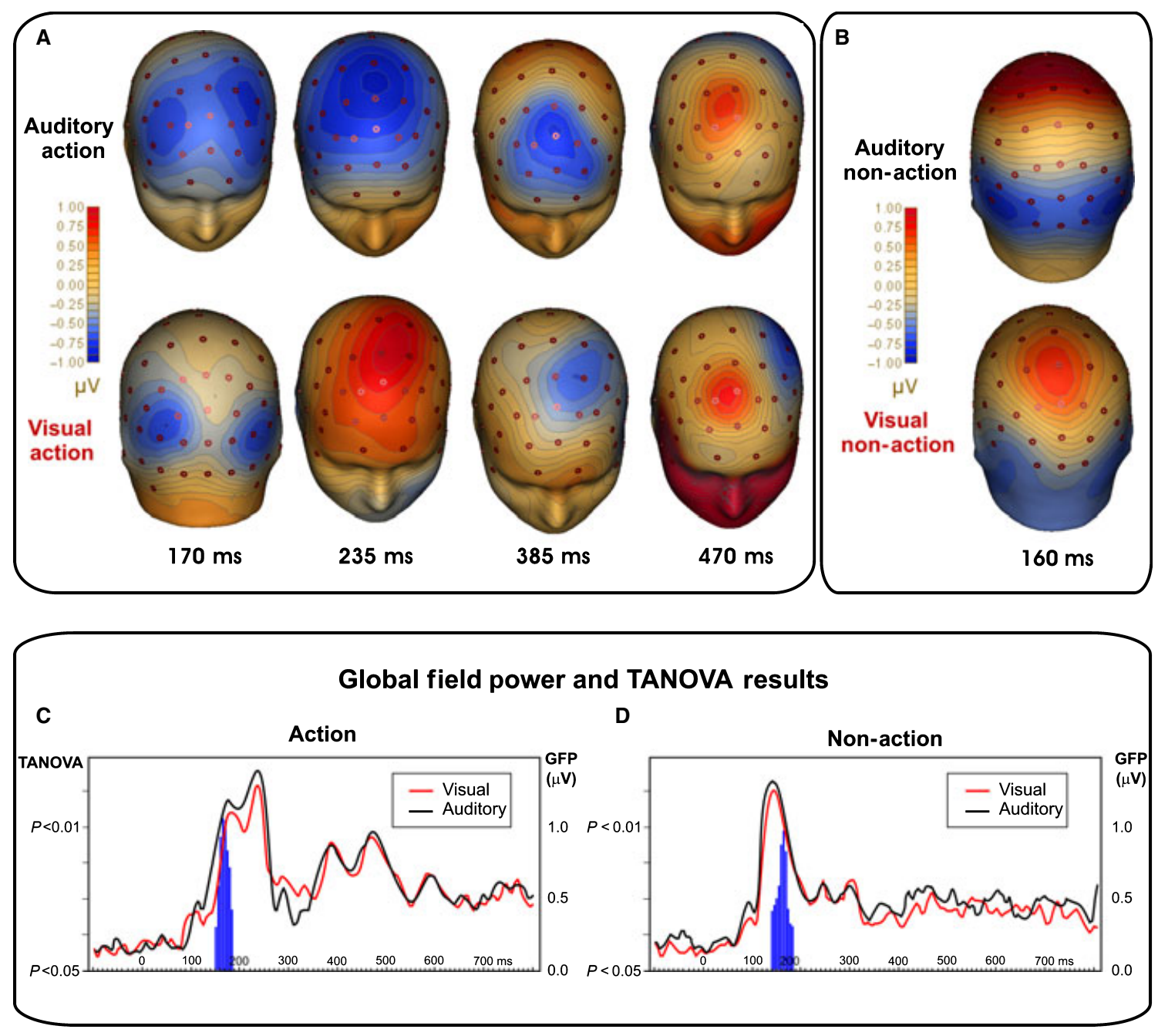

FIG. 4. (A) Topographic 3-D maps for action-related stimuli at peak latency for the four differential ERP components in the auditory and visual modalities; (B) topographic 3-D maps for non-action-related stimuli at $160 \mathrm{~ms}$ in both modalities; (C) global field power time course and TANOvA results (vertical bars) for action-related stimuli in visual and auditory modalities; and (D) the same data for non-action-related stimuli.

was similar to the timing for the early sensorial components before the significant effect that occurred at $170 \mathrm{~ms}$.

For both modalities, the component that peaked at $235 \mathrm{~ms}$ was localized in the left temporal lobe close to the STG. This source showed an onset at $175 \mathrm{~ms}$ for the auditory modality and $190 \mathrm{~ms}$ for the visual modality. For both modalities, the component that peaked at $385 \mathrm{~ms}$ was localized in the left frontal lobe of the precentral gyrus. This source showed an onset at $335 \mathrm{~ms}$ for the auditory modality and $350 \mathrm{~ms}$ for the visual modality. Finally, for both modalities, the component that peaked at $470 \mathrm{~ms}$ was localized to the left parietal lobe, where, in the auditory condition, the source was located in the superior parietal lobule and, in the visual condition, in the inferior parietal lobule. The onset of this source occurred at $395 \mathrm{~ms}$ for the auditory modality and at $410 \mathrm{~ms}$ for the visual modality.

Figure 6B shows the results of the spatiotemporal source models for the non-action-related conditions. For the auditory modality, at $160 \mathrm{~ms}$, the component was localized bilaterally in the temporal lobe in the anterior part of the STG. For the visual modality, the activity at the same latency was localized in the bilateral occipital lobe in the middle occipital gyrus. The time course of these sources (averaged over hemispheres) was similar, with an onset at $110 \mathrm{~ms}$.

\section{Discussion}

We predicted that visual and auditory stimuli that are related to human actions are processed differently from non-action-related stimuli. Crucially, the present experiment strongly supports previous observations (Pizzamiglio et al., 2005; Gazzola et al., 2006; Galati et al., 2008; De Lucia et al., 2009) that both the ERPs and fMRI activities produced by action- and non-action-related stimuli are clearly distinct. Following early activity (at approximately $170 \mathrm{~ms}$ ), which was related almost certainly to the sensory analysis of auditory and visual stimuli, a common set of activities was detected in the temporal, frontal and parietal regions of the left hemisphere during the processing of actionrelated information. In contrast, activity associated with non-actionrelated auditory and visual stimuli was observed at a different latency $(160 \mathrm{~ms})$ and was located bilaterally in the auditory and the visual cortex, respectively.

Therefore, the data described herein demonstrate, for the first time, the existence of shared ERPs following both auditory and visual stimulation. Given that the video clips used depicted the same actions that were represented by the sound clips, the strong overlap in activities might be due to the underlying nature of the action, which was identical but expressed in different modalities. A similar 


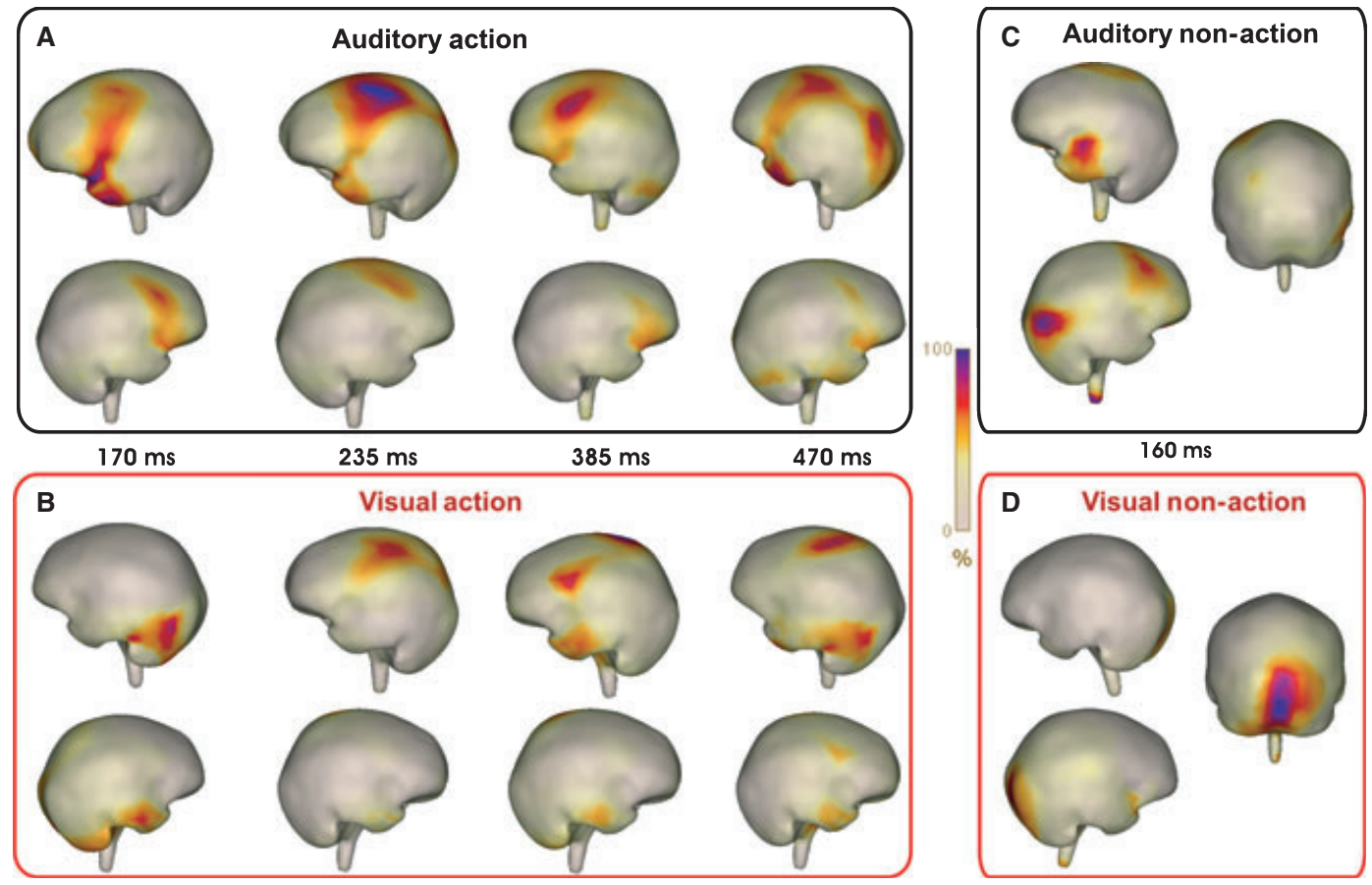

FIG. 5. Noise-normalized minimum-norm source estimations at peak latency for the significant repetition suppression effects (difference waves). (A, B) Action- and (C, D) non-action-related conditions for both auditory and visual modalities. All inverse solutions were scaled linearly between 0 and $100 \%$ to illustrate the spatial distributions of the estimates. Note that auditory and visual action-related stimuli produced similar source estimations for activity at 235,385 and 470 ms, which confirms the TANOVA results.

semantically based explanation was used to account for the anterior premotor activity in a previous fMRI action-sound experiment (Gazzola et al., 2006) and the association between sound comprehension and mouth/hand apraxic impairment in brain-damaged patients (Pazzaglia et al., 2008). The present data can also be used to gain insight into the dynamics and, in particular, the sequential organization of the perceptual and motor representations that occur in response to multimodal stimulations. For both modalities, when the timing of the ERPs responses was combined with their localization, a peak was identified at $235 \mathrm{~ms}$ in the STG, followed by a second peak at $385 \mathrm{~ms}$ in the left premotor area, and finally a third peak at $470 \mathrm{~ms}$ in the prefrontal and temporo-parietal area of the left hemisphere.

This clear sequence of events might help to clarify the role of temporal structures with regard to the competing interpretations that were described in the Introduction. In this respect, the first stage in interpreting the meaning of visual and auditory stimuli might take place in the temporal areas (Gazzola et al., 2006; Senkowski et al., 2007). This information is transmitted subsequently to the premotor area where the meaning is analysed further and a representation of the motor plan for the corresponding action is activated (Galati et al., 2008).

As mentioned above, the activity at $235 \mathrm{~ms}$ was localized within the STG for both auditory and visual action stimuli, even though it shows polarity inversion across the two modalities. A possible explanation is that the generators are located very close to each other but positioned differently within the folds of the cortex. To support this hypothesis, the data on Fig. 6A (right panels, second line) show that the dipoles representing the activity at $235 \mathrm{~ms}$ have similar locations in the two modalities (blue spheres), but their orientations are almost reversed, yielding similar topographies but with opposed polarity.

The left temporo-parietal activities that were observed following a longer delay might reflect 'the auditory and visual consequences of the executed action' (Iacoboni et al., 2001). In particular, we confirm that the parietal lobule might be also associated with the MNS, which is in agreement with previous studies that reported that this area is recruited during both the observation and the imitation of hand movements (Iacoboni et al., 1999; Molnar-Szakacs et al., 2005). Therefore, this parietal activity might be an indication that this part of the brain is involved in some type of motor preparation. In this regard, Buccino et al. (2004) proposed that the parietal cortex seems to be involved in the observation of an action to be imitated.

The spatial resolution of ERPs is notorious low and source localization techniques are just models of the possible underlying cortical activity. However, the above speculations, which derive from the mirror neuron hypothesis, fit well with the theory but also add pertinent insight regarding the respective roles of the different structures involved in the network. However, we accept that the present analysis does not demonstrate explicitly that the identified set of activities is associated definitely with mirror neurons. Nevertheless, it should be noted that, in the present study, the activated areas matched completely with other observations regarding the MNS in humans, in which similar fronto-parietal areas have been shown to be recruited in a variety of paradigms and are thought to be part of an action observation/execution matching system that mediates action understanding (Iacoboni et al., 1999; Aziz-Zadeh et al., 2004; Molnar-Szakacs et al., 2005; Pizzamiglio et al., 2005; Gazzola et al., 2006; Galati et al., 2008).

A final comment concerns the predominantly left brain lateralization that we observed. It has been proposed that this hemispheric asymmetry might be linked to the role of the MNS as a precursor to the development of the language area (Aziz-Zadeh et al., 2004). In contrast, other studies (Binkofsky et al., 1999; Buccino et al., 2001; Molnar-Szakacs et al., 2005; Aziz-Zadeh et al., 2006) have shown that there is bilateral involvement with regard to multimodal actionrelated stimuli in which the right hemisphere is recruited specifically for the purpose of processing visual action-related stimuli. However, 
Source time course
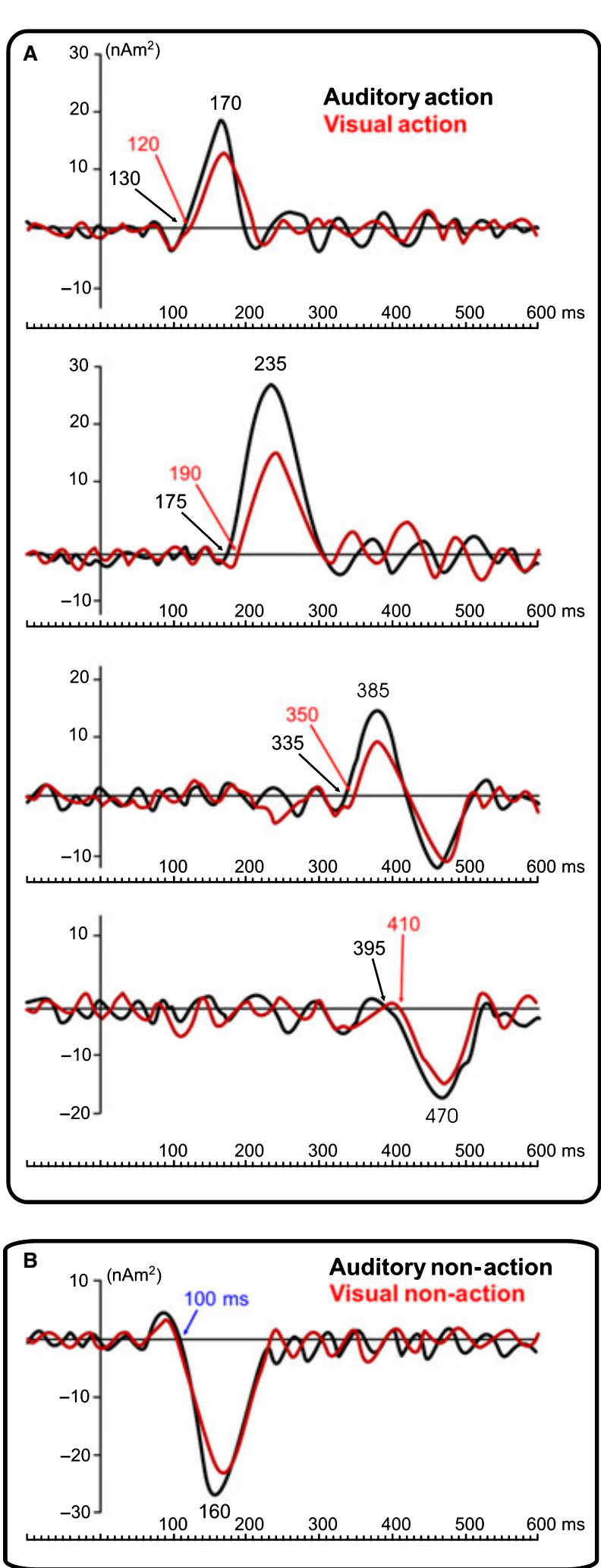

\section{Source localization}
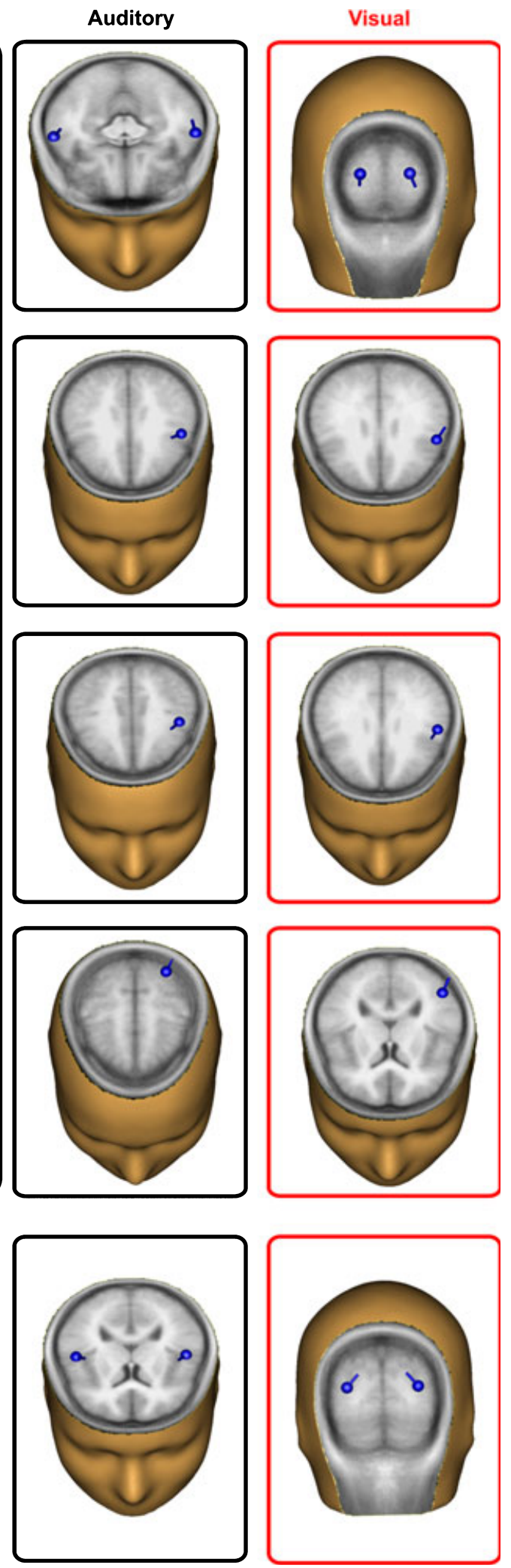

FIG. 6. (A) BESA spatio-temporal source models for the action-related conditions (difference waves). Left panel shows the time course of the cortical sources located in the right panels as projected on the 3-D MRI template. Black tracks represent processing of auditory stimuli and red (dashed) tracks represent processing of visual stimuli; these confirm the similar timing in the two modalities. Onset and peak latencies of the cerebral sources are labelled. Right panel shows the location (sphere) and the orientation (sphere vector) of the modelled sources. (B) As above, but for non-action-related conditions. 
it should be emphasized that the latter studies used either transcranic magnetic stimulation or fMRI techniques, whereas studies that have employed ERPs, including the present one, have consistently indicated the existence of left lateralized activity. In fact, we found only minimal right hemisphere activity in the visual task. It should also be noted that when techniques such as fMRI are used, static cerebral activation is displayed. In contrast, the ERPs employed in the present study possessed high temporal resolution and were able to indicate both the timing and the onset of activities that initiated in the left hemisphere and subsequently spread to the right. Ideomotor apraxia is a syndrome that provides an ideal model for understanding the role of the left hemisphere more fully. Interestingly, this disorder is associated with left frontal-temporal-parietal lesions that impair the transformation of motor representations into motor actions (Wheaton \& Hallett, 2007). Furthermore, a recent study (Pazzaglia et al., 2008) has shown that apraxic patients are unable to discriminate correct gestures from erroneous ones in action recognition tasks. The location and effects of these lesions appear to demonstrate a link between apraxia and the MNS, which supports the data with regard to the predominance of the left hemisphere and therefore also corroborates the relevance of MNS for understanding the evolution/ development of language.

In conclusion, our findings are consistent with the notion that action-related stimuli (visual and auditory) are processed in similar areas of brain, which supports the notion that MNS performs a multimodal function in humans. Moreover, the spatio-temporal analysis using ERPs showed that this processing was distributed widely in the left hemisphere and involved a larger brain network than was previously thought.

\section{Supporting Information}

Additional supporting information may be found in the online version of this article:

Table S1. Complete list of prime-target pairs used in the experimental design

Please note: As a service to our authors and readers, this journal provides supporting information supplied by the authors. Such materials are peer-reviewed and may be re-organized for online delivery, but are not copy-edited or typeset by Wiley-Blackwell. Technical support issues arising from supporting information (other than missing files) should be addressed to the authors.

\section{Abbreviations}

BESA, brain electrical source analysis; EEG, electroencephalogram; ERPs, event-related potentials; fMRI, functional magnetic resonance imaging; GFP, global field power; MNE, minimum norm estimate; MNS, mirror neuron system; MTG, mid-temporal gyrus; ROT, residual orthogonality tests; RSP, repetition suppression paradigm; RV, residual variance; SOT, source wave orthogonality test; STG, superior temporal gyrus; TANOVA, topographic analysis of variance.

\section{References}

Aziz-Zadeh, L., Iacoboni, M., Zaidel, E., Wilson, S. \& Mazziotta, J. (2004) Left hemisphere motor facilitation in response to manual action sounds. Eur. J. Neurosci., 19, 2609-2612.

Aziz-Zadeh, L., Koski, L., Zaidel, E., Mazziotta, J. \& Iacoboni, M. (2006) Lateralization of the mirror neuron system. J. Neurosci., 26, 2964-2970.

Binkofsky, F., Buccino, G., Posse, S., Seitz, R.J., Rizzolatti, G. \& Freund, H.J. (1999) A fronto-parietal circuit for object manipulation in man: evidence from an fMRI study. Eur. J. Neurosci., 11, 3276-3286.
Binkofsky, F., Amunts, K., Stephan, K.M., Posse, S., Schormann, T., Freund, H.J., Zilles, K. \& Seitz, R.J. (2000) Broca's region subserves imagery of motion: a combined cytoarchitectonic and fMRI study. Hum. Brain Mapp., 11, 273-285.

Bocker, K.B.E., Cornelis, H.M., Brunia, C.H.M. \& Van den Berg-Lenss, M.M.C. (1994) A spatiotemporal dipole model of the stimulus preceding negativity (SPN) prior to feedback stimuli. Brain Topogr., 7, 71-88.

Bruzzo, A., Borghi, A.M. \& Ghirlanda, S. (2008) Hand-object interaction in perspective. Neurosci. Lett., 441, 61-65.

Buccino, G., Binkofsky, F., Fink, G.R., Fadiga, L., Fogassi, L., Gallese, V., Seitz, R.J., Zilles, K., Rizzolatti, G. \& Freund, H.J. (2001) Action observation activates premotor and parietal areas in a somatotopic manner: an fMRI study. Eur.J. Neurosci., 13, 400-404.

Buccino, G., Binkofski, F. \& Raggio, L. (2004) The mirror neuron system and action recognition. Brain Lang., 89, 370-376.

Corballis, M.C. (2002) From Hand to Mouth: The Origins of Language. Princeton University Press, Princeton, NJ.

Dale, A.M. \& Sereno, M.I. (1993) Improved localization of cortical activity by combining EEG and MEG with MRI cortical surface reconstruction: a linear approach. J. Cogn. Neurosci., 5, 162-176.

De Lucia, M., Camen, C., Clarke, S. \& Murray, M.M. (2009) The role of actions in auditory object discrimination. Neuroimage, 48, 475-485.

Decety, J., Grèzes, J., Costes, N., Perani, D., Jeannerod, M., Procyk, E., Grassi, F. \& Fazio, F. (1997) Brain activity during observation of actions. Influence of action content and subject's strategy. Brain, 12, 1763-1777.

Di Russo, F., Martinez, A., Sereno, M.I., Pitzalis, S. \& Hilyard, S.A. (2002) The cortical sources of the early components of the visual evoked potential. Hum. Brain Mapp., 15, 95-111.

Galati, G., Committeri, G., Spitoni, G., Aprile, T., Di Russo, F., Pitzalis, S. \& Pizzamiglio, L. (2008) A selective representation of the meaning of actions in the auditory mirror system. Neuroimage, 40, 1274-1286.

Gallese, V., Fadiga, L., Fogassi, L. \& Rizzolatti, G. (1996) Action recognition in the premotor cortex. Brain, 119, 593-609.

Gazzola, V., Aziz-Zadeh, L. \& Keysers, C. (2006) Empathy and somatotopic auditory mirror system in humans. Curr. Biol., 16, 1824-1829.

Grafton, S.T., Arbib, M.A., Fadiga, L. \& Rizzolatti, G. (1996) Localization of grasp representations in humans by positron emission tomography. 2. Observation compared with imagination. Exp. Brain Res., 112, 103111.

Grill-Spector, K., Henson, R. \& Martin, A. (2006) Repetition and the brain: neural models of stimulus-specific effects. Trends Cogn. Sci., 10, $14-23$.

Gruber, T., Giabbiconi, C.M., Trujillo-Barreto, N.J. \& Müller, M.M. (2006) Repetition suppression of induced gamma-band responses is eliminated by task switching. Eur. J. Neurosci., 24, 2654-2660.

Hämäläinen, M.S. \& Ilmoniemi, R.J. (1984) Interpreting Measured Magnetic Fields of the Brain: Minimum Norm Estimates of Current Distributions. Helsinki University of Technology, Technical Report TKK-F-A559.

Heiser, M., Iacoboni, M., Maeda, F., Marcus, J. \& Mazziotta, J.C. (2003) The essential role of Broca's area in imitation. Eur. J. Neurosci., 17, 1123-1128.

Henson, R.N. (2003) Neuroimaging studies of priming. Prog. Neurobiol., 70, 53-81.

Hickok, G. (2009) Eight problems for the mirror neuron theory of action understanding in monkeys and humans. J. Cogn. Neurosci., 21, 12291243 .

Iacoboni, M. \& Dapretto, M. (2006) The mirror neuron system and the consequences of its dysfunction. Nat. Rev. Neurosci., 7, 942-951.

Iacoboni, M., Woods, R.P., Brass, M., Bekkering, H., Mazziotta, J.C. \& Rizzolatti, G. (1999) Cortical mechanisms of human imitation. Science, 24, 2526-2528.

Iacoboni, M., Koski, L.M., Brass, M., Bekkering, H., Woods, R.P., Dubeau, M.C., Mazziotta, J.C. \& Rizzolatti, G. (2001) Reafferent copies of imitated actions in the right superior temporal cortex. Proc. Natl Acad. Sci. USA, 20, 13995-13999.

Ilmoniemi, R.J. (1993) Models of source currents in the brain. Brain Topogr., 5, 331-336.

Keysers, C., Kohler, E., Umiltà, M.A., Fogassi, L. \& Gallese, V. (2003) Audiovisual mirror neurons and action recognition. Exp. Brain Res., 153, 628-636.

Kilner, J.M., Vargas, C., Duval, S., Blakemore, S.J. \& Sirigu, A. (2004) Motor activation prior to observation of a predicted movement. Nat. Neurosci., 7, 299-1301.

Kohler, E., Keysers, C., Umiltà, M.A., Fogassi, L., Gallese, V. \& Rizzolatti, G. (2002) Hearing sounds, understanding actions: action representation in mirror neurons. Science, 297, 846-848. 
Lehmann, D. 1987. Principles of spatial analysis. In Gevins, A.S. \& Remond, A. (Eds), Methods of Analysis of Brain Electrical and Magnetic Signals. Handbook of Electroencephalography and Clinical Neurophysiology. Vol. 1. Elsevier, Amsterdam, pp. 309-354.

Lehmann, D. \& Skrandies, W. (1980) Reference-free identification of components of checkerboard-evoked multichannel potential fields. Electroencephalogr. Clin. Neurophysiol., 48, 609-621.

Lin, F.H., Witzel, T., Ahlfors, S.P., Stufflebeam, S.M., Belliveau, J.W. \& Hämäläinen, M.S. (2006) Assessing and improving the spatial accuracy in MEG source localization by depth-weighted minimum-norm estimates. Neuroimage, 31, 160-171.

Lingnau, A., Gesierich, B. \& Caramazza, A. (2009) Asymmetric fMRI adaptation reveals no evidence for mirror neurons in humans. Proc. Natl Acad. Sci. USA, 106, 9925-9930.

Molnar-Szakacs, I., Iacoboni, M., Koski, L. \& Mazziotta, J.C. (2005) Functional segregation within pars opercularis of the inferior frontal gyrus: evidence from fMRI studies of imitation and action observation. Cereb. Cortex, 15, 986-994.

Murray, M.M., Brunet, D. \& Michel, C.M. (2008) Topographic ERP analyses: a step-by-step tutorial review. Brain Topogr., 20, 249-264.

Noppeney, U. \& Penny, W.D. (2006) Two approaches to repetition suppression. Hum. Brain Mapp., 27, 411-416.
Pazzaglia, M., Pizzamiglio, L., Pes, E. \& Aglioti, S.M. (2008) The sound of actions in apraxia. Curr. Biol., 18, 1766-1772.

Pizzamiglio, L., Aprile, T., Spitoni, G., Pitzalis, S., Bates, E., D’Amico, S. \& Di Russo, F. (2005) Separate neural systems for processing action and nonaction-related sounds. Neuroimage, 24, 852-861.

Rizzolatti, G., Fadiga, L., Gallese, V. \& Fogassi, L. (1996) Premotor cortex and the recognition of motor actions. Cogn. Brain Res., 3, 131-141.

Senkowski, D., Saint-Amour, D., Kelly, S.P. \& Foxe, J.J. (2007) Multisensory processing of naturalistic objects in motion: a high-density electrical mapping and source estimation study. Neuroimage, 36, 377-388.

Teder-Sälejärvi, W.A., McDonald, J.J., Di Russo, F. \& Hillyard, S.A. (2002) An analysis of audio-visual crossmodal integration by means of event-related potential (ERP) recordings. Cogn. Brain Res., 14, 106114

Tettamanti, M., Buccino, G., Saccuman, M.C., Gallese, V., Danna, M., Scifo, P., Rizzolatti, G., Cappa, S.F. \& Perani, D. (2005) Listening to action-related sentences activates fronto-parietal motor circuits. J. Cogn. Neurosci., 17, 273-281.

Thierry, G., Giraud, A.L. \& Price, C. (2003) Hemispheric dissociation in access to the human semantic system. Neuron, 38, 499-506.

Wheaton, L.A. \& Hallett, M. (2007) Ideomotor apraxia: a review. J. Neurol. Sci., 15, 1-10. 